pleta ocorreu em 12,3\% das pacientes, já a resposta patológica completa aconteceu em 10,3\% dos casos. Conclusões: observamos uma correlação estatisticamente significante entre o número de ciclos e a resposta a quimioterapia primária. As pacientes, que receberam 4 ciclos tiveram melhor resposta em comparação com as que receberam 3 ciclos. Também verificamos uma concordância estatisticamente significativa entre a avaliação pelo exame clínico da resposta à quimioterapia primária e o achado anatomopatológico. Não foram observadas correlações estatisticamente significantes entre as demais variáveis

PALAVRAS-CHAVES: Mamas - Câncer - Tratamento - Quimioterapia Primária - Neoadjuvante

\title{
Efeitos da exposição à fumaça de cigarro na performance reprodutiva, no sistema antioxidante e no metabolismo de ratas diabéticas
}

\section{Effects of exposure to cigarette smoke on the reproductive performance, antioxidant system and metabolism of diabetic female rats}

Autor: Maricelma da Silva Soares de Souza

Orientadora: Profa. Dra. Débora Cristina Damasceno

Tese apresentada ao Programa de Pós-Graduação em Ginecologia, Obstetrícia e Mastologia da Faculdade de Medicina de Botucatu - UNESP- para obtenção do título de Doutor, em 16 de dezembro de 2005.

Objetivo: avaliar o efeito do diabete grave e da exposição à fumaça de cigarro de tabaco sobre a performance reprodutiva, desenvolvimento fetal e placentário, sistema antioxidante e no metabolismo de ratas Wistar prenhes. Material e Método: as ratas foram expostas à fumaça de 10 cigarros, por 30 minutos, duas vezes ao dia, antes e durante o período de prenhez. O diabete foi induzido por streptozotocin (40 mg/kg; i.v). Foram determinados quatro grupos de acordo com a presença ou não do diabete e a exposição ou não à fumaça. No $21^{\circ}$ dia de prenhez, as ratas foram anestesiadas e mortas por dessangramento para coleta de sangue para determinações das atividades enzimáticas da superóxido dismutase e glutationa peroxidase e concentrações de malonaldeído, glutationa total, triglicérides, colesterol e proteínas totais. Os cornos uterinos foram expostos para análise dos parâmetros de performance reprodutiva. Os recém-nascidos foram classificados com peso adequado (AIP), pequeno (PIP) e grande (GIP) para idade de prenhez. Resultados: as ratas com diabete grave apresentaram hiperglicemia, hipercolesterolemia e hipertrigliceridemia. O diabete grave e a exposição à fumaça de cigarro, isoladamente, não mostraram diferenças significativas quanto aos parâmetros da performance reprodutiva, mas causaram restrição de crescimento intra-uterino (RCIU). A associação do diabete grave e da exposição à fumaça de cigarro causou comprometimento da performance reprodutiva materna e disfunções placentárias, sendo estas confirmadas pela placentomegalia, aumento do índice placentário e RCIU, além de induzir os componentes do sistema antioxidante. Embora não tenha sido capaz de conter a peroxidação lipídica, estas ratas apresentaram também hipertrigliceridemia. A presença de hipertrigliceridemia no grupo de ratas diabéticas expostas à fumaça foi decorrente do diabete grave e não da exposição à fumaça de cigarro.

PALAVRAS-CHAVE: Diabete melito; Tabagismo; Cigarro; Performance reprodutiva; Sistema antioxidante; Metabolismo lipídico

Resumo de Tese

\section{Detecção do linfonodo sentinela com ${ }^{99 m}$ Tc-fitato em pacientes com carcinoma invasor do colo uterino}

Autor: Lucas Barbosa da Silva

Orientador: Prof.Dr. Paulo Traiman

Co-orientador: Dr. Agnaldo Lopes da Silva Filho

Tese apresentada ao Programa de Pós-Graduação em Ginecologia, Obstetrícia e Mastologia da Faculdade de Medicina de Botucatu - UNESP, para obtenção do título de Doutor, em 9 de dezembro de 2004.

\section{Resumo}

Objetivo: investigar a viabilidade da detecção do linfonodo sentinela (LNS) utilizando o mapeamento linfático radioisotópico com ${ }^{99 \mathrm{~m}-}$ tecnécio-fitato em pacien- tes submetidas a histerectomia radical e linfadenectomia pélvica para tratamento de câncer de colo uterino. Método: entre julho de 2001 e fevereiro de 2003, 56 pacientes com câncer de colo uterino estádi- 